\title{
Apatinib plus S-1 for previously treated, advanced gastric or gastro-oesophageal junction adenocarcinoma: a phase 2, single-arm, prospective study
}

\author{
Chao Jing ${ }^{1}$, Zhigang Bai ${ }^{1}$, Jun Zhang ${ }^{1}$, Hongpeng Jiang ${ }^{1}$, Kai Pang ${ }^{1}$, Xiaobao Yang ${ }^{1}$, Shu \\ Yan $^{1}$, Jie Yin ${ }^{1}$, Jun Cai ${ }^{1}$, Zhongtao Zhang ${ }^{1}$, and Wei Deng ${ }^{1}$ \\ ${ }^{1}$ Capital Medical University Affiliated Beijing Friendship Hospital
}

July 24, 2020

\begin{abstract}
Aim: The current management of advanced gastric or gastro-oesophageal junction adenocarcinoma remains unsatisfactory. We investigated the efficacy and safety of the combination therapy of apatinib and S-1, considering the potential advantage of home-based treatment without hospital admission, in patients with platinum-refractory gastric or gastro-oesophageal junction adenocarcinoma. Methods:Between April 2015 and May 2019, we included 37 patients with advanced gastric or gastrooesophageal junction adenocarcinoma refractory to first-line platinum-containing therapy, who were treated with apatinib at an initial dose of $500 \mathrm{mg}$ once daily continuously and S-1 at a dose of 40-60 mg twice daily on days 1-14 of a 21-day cycle. The primary endpoints were progression-free survival (PFS) and overall survival (OS). The secondary endpoints were objective response rates, disease control rates, and safety. Results:At the data cutoff, the median PFS and OS were 4.2 months and 8.2 months, respectively. Of 37 eligible patients, $8(21.6 \%)$ patients reached objective responses, $31(83.8 \%)$ patients reached disease control. Grade 3 or 4 adverse events occurred in 8 (21.6\%) patients, including hand-foot syndrome, hypertension, and diarrhea, etc. Conclusions: The combination of Apatinib and S-1 showed promising efficacy and manageable toxicity as a home-based, second-line therapy in patients with advanced gastric or gastro-oesophageal junction adenocarcinoma refractory to platinum-containing therapy.
\end{abstract}

\section{Indroduction}

Based on the Global Cancer Statistics [1], gastric cancer is the fifth most commonly diagnosed cancer and is the third leading cause of cancer deaths. In 2018, there were more than 1 million new cases and approximate 783,000 deaths because of gastric cancer. East Asia is the most heavily affected region from gastric cancer. The incidence is usually lower in the Americas and Northern Europe [2].

Screening and early diagnosis play a key role in reducing the mortality resulting from gastric cancer. With the increased use of gastroscopy and the development of other screening methods, East Asian countries such as Japan and Korea currently show much lower mortality rates [3]. However, because of the limitation of skills of endoscopic physicians and the low availability of gastroscopy, universal screening has not been used in many other countries $[4,5]$. China lags in comprehensive gastric cancer screening, and $80 \%$ of gastric cancer patients are already in late-stages when diagnosed [6]. In addition, nearly one-third gastric cancer patients in China who underwent adjuvant capecitabine plus oxaliplatin after D2 gastrectomy, either relapsed, or developed a new gastric cancer, and died, within 5 years of treatment [7]. According to the National Comprehensive Cancer Network (NCCN) [8] and Chinese Society of Clinical Oncology (CSCO) [9] guidelines, there are only limited choices for second-line treatment, and the treatment of recurrent or unresectable advanced gastric cancer is not highly effective. Therefore, we aimed to explore a second-line therapy in gastric cancer patients after the failure of platinum-containing treatments. 
With intensive research on tumor angiogenesis, it was discovered that small-molecule vascular endothelial growth factor receptor tyrosine kinase inhibitors (VEFGR-TKIs) could significantly enhance the efficacy of chemotherapy [10]. Apatinib, an oral small molecule VEGFR-TKI, inhibited the tyrosine kinase activity of VEGFR-2 with high selectivity, thereby strongly inhibiting tumor angiogenesis [11]. Apatinib has been approved by the China Food and Drug Administration (CFDA) as a monotherapy for patients with advanced gastric or gastric-esophageal junction adenocarcinoma refractory to at least two chemotherapy regimens, since December 13, 2014, which opened a new chapter in the targeted therapy of advanced gastric cancer after Trastuzumab in China. Several clinical studies have proved its efficacy and safety in third-line treatment of advanced gastric cancer $[12,13]$. S-1 is a fluorouracil-derived combination anticancer agent consisting of tegafur (FT), gimeracil (CDHP) and oteracil (OXO) [14]. Numerous studies $[15,16]$ demonstrated that S-1 was suitable for adjuvant, first-line, and second-line chemotherapy for gastric cancer. Both apatinib and S-1 are among the most commonly used oral drugs for gastric cancer in clinical practice in China [9] , and, it was found in cytological experiments that the combination of apatinib with 5-FU was synergistic for advanced gastric cancer[17]. Therefore, we aimed to explore the combined effects of apatinib plus S-1 through this phase-2 trial. Recently with the emergence of novel coronavirus (COVID-19) pneumonia, there is a serious scarcity of medical resources worldwide and limited outdoor activities are being recommended for cancer patients by various cancer societies including European Society for Medical Oncology (ESMO) [18]. Therefore, it might be worthwhile to evaluate substitution of intravenous agents by oral drugs. Considering the global outbreak of the novel coronavirus and the possibility of its long-term existence, oral administration of apatinib combined with S-1 might be a preferred option for patients because it can potentially minimize visits and hospitalization, limiting the spread of COVID-19.

Therefore, for patients with advanced gastric or gastro-oesophageal junction adenocarcinoma refractory to first-line platinum-containing chemotherapy, the effectiveness and safety of apatinib combined with S-1 needs exploration, and as both drugs are taken orally, this might increase the available treatment options for elderly patients, or those who refuse intravenous treatment, or those in a special situation.

\section{Methods}

\section{Patient characteristics}

We conducted this phase 2, single-arm, prospective study at the Beijing Friendship Hospital affiliated to Capital Medical University between April 2015 and May 2019. Eligible patients were aged 18 years or older; had unresectable advanced or recurrent gastric or gastro-oesophageal junction cancer histologically confirmed to be adenocarcinoma; had a measurable tumor which progressed during the first-line platinum-containing (XELOX or FOLFOX regimen, which includes oxaliplatin plus capecitabine or fluorouracil plus oxaliplatin respectively) treatment, or within 6 months after last platinum-containing adjuvant chemotherapy; had Eastern Cooperative Oncology Group (ECOG) performance status score of $0 \sim 2$ and a life expectancy of at least 3 months; had adequate organ function. Exclusion criteria were: previous application of apatinib or other TKIs; uncontrollable hypertension; various factors affecting oral drug absorption (such as severe dysphagia, severe vomiting, chronic diarrhea, and obstruction of digestive tract etc.); serious heart and lung dysfunction; neurological and mental illness. The full inclusion and exclusion criteria are shown in the appendix.

The trial was discontinued in selected patients for the following reasons: when tumor progressed, or serious adverse events occurred, or the patients requested withdrawal, or the investigator determined that there was a need to discontinue the trial.

\section{Ethical committee Clearance}

The trial was approved by the Research Ethics Committee of the Beijing Friendship Hospital affiliated to Capital Medical University and was done in accordance with the Declaration of Helsinki. All patients provided written informed consent. This trial was registered with ClinicalTrials.gov, number NCT04338438.

\section{Procedures}


Patients received apatinib at an initial dose of $500 \mathrm{mg}$ once daily continuously, plus S-1 at a dose of 40-60 $\mathrm{mg}$ twice daily (40-60 mg twice daily dose depending on the patient's body surface area: $40 \mathrm{mg}$ if $<1.25 \mathrm{~m}^{2}$; $50 \mathrm{mg}$ if $1.25-1.5 \mathrm{~m}^{2}$; and $60 \mathrm{mg}$ if $>1.5 \mathrm{~m}^{2}$ ) on days $1-14$ of a 21-day cycle. Medication was continued until the disease progression, withdrawal requirement, or intolerable adverse events.

Before treatment initiation, we used abdominal enhanced CT/MRI to measure and document the measurable lesions. During the treatment period, patients visited the outpatient department every three weeks for assessment that included: Vital signs, blood routine examination, blood biochemical examination, urine routine test, 12-lead ECG, etc. Adverse events were graded according to the National Cancer Institute Common Terminology Criteria for Adverse Events (NCI-CTCAE, version 4.03) and monitored continuously during the course of treatment. In addition, according to the Response Evaluation Criteria In Solid Tumors (RECIST, version 1.1) [19], every 6 weeks the investigators evaluated the treatment response through enhanced CT/MRI of abdomen and pelvis and metastatic sites, and tumor markers (including CEA, CA 125, CA 199, AFP, and NSE) until the experiment was discontinued. The best response should be kept at least 4 weeks for confirmation.

For the management of adverse events, dose adjustments were permitted for both, apatinib and S-1, including drug suspension and dose reduction. For apatinib, if patients had grade 4 hematologic adverse events, treatment was suspended until recovery to grade 1 or below, and, then restarted with the reduced dose. If the patients had grade 3 hematologic adverse events or grade 3-4 non-hematologic adverse events, at the first instance, apatinib was suspended until recovery to grade 1 or below, and, then restarted with the nonreduced dose. At the second instance of the grade 3 hematologic adverse events or grade 3-4 non-hematologic adverse events, the subsequent treatment with apatinib was administered using the reduced dose. For S-1, if patients had grade 3-4 hematologic adverse events or grade 3-4 non-hematologic adverse events (except hypertension, hand-foot syndrome, and proteinuria), it was suspended until recovery to grade 1 or below, and, then the single dose was reduced from $60 \mathrm{mg}$ to $50 \mathrm{mg}$, or from $50 \mathrm{mg}$ to $40 \mathrm{mg}$ twice daily, as applicable, and no further dose re-escalation was allowed [13].

\section{Outcomes}

The primary endpoints were progression-free survival (PFS) and overall survival (OS). Progression-free survival referred to the duration from the beginning of the treatment to the disease progression, or death from any cause, or last progression-free survival assessment for patients alive without progression. Overall survival was defined as the interval from the enrollment to death from any cause. The secondary endpoints were the objective response rates (ORR), and disease control rates (DCR) (according to RECIST 1.1), and safety. The ORR was defined as the proportion of patients with measurable disease who achieved complete or partial response. The DCR was defined as the proportion of patients achieving stable disease or complete response or partial response.

\section{Statistical Analysis}

Using the Exact single-stage phase- 2 design, with a power of $90 \%$ and a type I error of $5 \%$ the study detected an improvement in the 6-mouth progression-free survival from previous controls of about 18\%-36\%[20,21]. We initially expected the 6-month progression-free survival would be $40 \%$, and under these assumptions, 36 or more patients should be enrolled. Eventually, if the 6-month progression-free survival reached $30.5 \%$, the treatment regimen would be considered success.

The time of data cut-off was set at Jan 31, 2020. The full analysis set (FAS) consisted of intent-to-treat (ITT) patients, including all patients who were followed up after enrollment. The per-protocol set (PPS) was a subset of FAS. Patients were included in PPS when they discontinued the study due to progressive disease or intolerant adverse events or death. Patients in PPS received at least 2 cycles of treatment, with good compliance and adherence to the protocol, and had a complete baseline and post-treatment evaluation. Because sufficient compliance and complete records were found for all enrolled patients, the efficacy were only analyzed for ITT. Safety set (SS) consisted of patients with at least one treatment and safety data and all patients enrolled in this study were included in the safety data set. 
We calculated the proportions of patients with different treatment responses and prognoses, and we assessed 95\% CIs using Wilson procedure. We assessed the median PFS and OS and their 95\%CIs using the KaplanMeier Model. In addition, we also used multiple Kaplan-Meier Model to assess whether there were significant differences in median PFS or median OS between different subgroups, such as patients with recurrent tumors, or with unresectable tumors. All statistical analyses were two-sided and significance was set at $\mathrm{P}<0.05$. The software used for all statistical analyses was SPSS Statistics (version 25.0).

\section{Results}

\section{Patient Characteristics}

Between April 2015 and May 2019, 39 patients were screened in this all-oral treatment plan, 37 of whom were enrolled in this study. One patient was excluded due to previous treatment with oxaliplatin plus S-1, and another patient was excluded because of pre-existing severe vomiting that might affect oral drug intake. The patients' baseline information is displayed in Table 1.

At the time of data collection (Jan 31, 2020), treatment was discontinued in 35 patients, while 2 patients were still on treatment. Twenty-nine patients eventually discontinued the trial because of disease progression, 4 patients discontinued the trial because of intolerable adverse events, while 2 patients discontinued due to consent withdrawal (Figure 1). The median follow-up duration for the cohort $(\mathrm{n}=37)$ was 8.4 months.

\section{Efficacy}

At the end of the follow-up, $26(70.3 \%)$ patients had died, and $11(29.7 \%)$ patients were still alive. As for the primary endpoints, the median PFS was 4.2 months (IQR 2.7-7.0; 95\%CI 3.50-4.90) and the median OS was 8.2 months (IQR 4.95-12.6; 95\%CI 4.69-11.71); 6-month progression-free survival was $31.5 \%$ (95\% CI 16-48); 9-month progression-free survival was $17.5 \%$ (95\% CI 6-33); 6-month overall survival was $62 \%$ (95\% CI 45-76); 12-month overall survival was $36 \%$ (95\%CI 20-51); 2-year overall survival was $21.7 \%$ (95\% CI 8-39). (Figure 2)

Among all patients with efficacy evaluation, $0(0 \%)$ of 37 patients achieved complete response, $8(21.6 \%)$ of 37 patients achieved partial response, $23(62.1 \%)$ of 37 patients reached stable disease, and $6(16.2 \%)$ patients achieved progressive disease after first post-baseline imaging evaluation (Figure 3). As for the secondary endpoints, objective response was achieved in 8 of 37 patients (ORR: 21.6\%; 95\%CI 10.4\%-38.7\%). Disease control was achieved in 31 of 37 patients (DCR: 83.8\%; 95\%CI 67.3\%-93.2\%).

In this study, we also observed that the patients with recurrent gastric or gastro-oesophageal junction adenocarcinoma had a significantly longer median OS than patients with radically unresectable disease (18.0 months vs. 6.0months; HR: 3.318; 95\% CI 1.362-8.085; $\mathrm{P}=0.005$ ) (Figure 4).

\section{Safety}

All 37 patients were included in the safety analysis. The overall incidence of any-grade adverse events was $100 \%$. Most events were graded 1 or 2 . The most common AEs were hypertension in 11 patients (29.7\%), hand-foot syndrome in 12 patients $(32.4 \%)$, diarrhea in 12 patients $(32.4 \%)$, elevated transaminase in 14 patients $(37.8 \%)$, leukopenia in 12 patients $(32.4 \%)$. Eight patients $(21.6 \%)$ had grade $3-4$ adverse events, including hand-foot syndrome, acute intestinal obstruction, upper gastrointestinal bleeding and severe anemia, hypertension, fatigue, diarrhea, leukopenia. Among the 8 patients, 4 patients discontinued this trial for AEs: one for serious fatigue, one for acute intestinal obstruction, one for upper gastrointestinal bleeding and severe anemia, and one for grade 4 hand-foot syndrome. No treatment-related deaths occurred. Details of adverse events are shown in the Table 2.

Eventually, there were 9 patients $(24.3 \%)$ who required dose reduction for apatinib, of whom 6 patients experienced only one dose reduction and 3 patients had two dose reduction. 2 patients had dose reduction during the first cycle. Dose reduction occurred in 6 patients (16.2\%) for S-1, among whom 4 patients required one dose reduction and 2 patients had two dose reduction. 
In the analysis of adverse events and efficacy, it was observed that patients with any grade hand-foot syndrome had a longer median PFS (8.2 months vs. 4.2 months; HR 0.451; 95\%CI 0.197-1.031; $\mathrm{P}=0.059$; log-rank $\mathrm{P}=0.043)$ and median $\mathrm{OS}(13.0$ months vs. 6.0 months; HR $0.445 ; 95 \% \mathrm{CI}$ 0.186-1.067; $\mathrm{P}=0.70$; $\log$-rank $\mathrm{P}=0.061)$. Furthermore, the median PFS of patients who had any grade proteinuria was longer than those who did not (5.6 months vs 4.2 months; HR 1.421; 95\%CI 0.632-3.194; $\mathrm{P}=0.395 ; \log$-rank $\mathrm{P}=0.369$ ).

\section{Discussion}

In this single-arm trial, we assessed the efficacy and safety of the oral combination of apatinib and S-1 for patients with unresectable, advanced gastric or gastro-oesophageal junction adenocarcinoma, or recurrent gastric or gastro-oesophageal junction adenocarcinoma as a home-based second-line therapy. In our study, the median progression-free survival was 4.2 months and the median overall survival was 8.2 months, 8 $(21.6 \%)$ patients reached objective response, and 31 (83.8\%) patients reached disease control.

To our knowledge, the effects of current second-line treatment schemes for advanced gastric cancer remain unsatisfactory. The WJOG 4007 Trial [22], a phase-3 study in Japan, enrolled 233 patients with advanced gastric cancer refractory to treatment with fluoropyrimidine plus platinum. The median PFS and the median OS of the paclitaxel group was 3.6 months and 9.5 months, respectively, whereas, the median PFS and the median OS in the irinotecan group was 2.3 months and 8.4 months, respectively. No difference in OS between paclitaxel and irinotecan groups was observed in that study. These two monotherapies became classical second-line treatments in advanced gastric cancer. In RAINBOW study [21], a phase 3 trial, Ramucirumab plus Paclitaxel showed a significant improvement in patients with previously treated advanced gastric or gastro-esophageal junction adenocarcinoma; the objective response rate was $28 \%$, the median PFS reached 4.4 months, and in Asian patients the median OS reached 12.1 months. This study established the combination of ramucirumab and paclitaxel as a leading second-line chemotherapy regimen for advanced gastric cancer. In recent years, with the attention to immunotherapy, the KEYNOTE-061 study [23], another phase 3 trial, showed that the median OS of patients treated with pembrolizumab monotherapy reached 9.1 months. However, compared with paclitaxel, pembrolizumab monotherapy did not significantly improve overall survival for PD-L1 positive advanced gastric or gastro-esophageal junction adenocarcinoma (combined positive score [CPS] of 1 or higher). With reference to other second-line chemotherapy regimens $[20,24]$, the median PFS and median OS in patients using apatinib plus S-1 in this study were comparable to the existing studies, and had advantages in the form of convenience and mild adverse reactions. It is believed that the combination of apatinib and S-1 would have a better performance after dose adjustment and toxicity management. Furthermore, the combined use of multiple types of drugs is a new trend, it can be expected that the triple-combination of immunotherapy with chemotherapy and targeted therapy may achieve the desired result [25].

The recommended dose of apatinib is 500-850mg once daily[12,13], but considering the patients ages and conditions and the combining usage of S-1, in our study apatinib was given at 500mg once daily and S1 at the standard dose. Even though, the incidence of any grade adverse events was 100\%. Most adverse events were graded 1 or 2 ; grade 3 or 4 adverse events were mostly manageable; and no treatment-related death occurred. The common adverse events included hematological adverse events, elevated transaminase, hypertension, diarrhea, hand-foot syndrome, and proteinuria, etc. Among these adverse events, we believe that hand-foot syndrome, hypertension, and proteinuria were because of antiangiogenic therapy; anorexia, abdominal pain, diarrhea, elevated transaminase, and myelosuppression were S-1-related adverse events. In the analysis of toxicity and efficacy, similar to previous studies [26,27], the occurrence of hand-foot syndrome and proteinuria may indicate a better treatment outcome of anti-angiogenesis therapy, however, it might be attributed to the small sample size, we did not observe the statistical significance in the assessment of toxicity and efficacy.

In subgroup analysis, the reasons for a better prognosis among patients with recurrent tumors may be as follows: Firstly, opposite to the uncurable lesions, the primary tumor has been resected completely, and patients have had a tumor-free period. Secondly, compared with the patients who received first- and secondline chemotherapy consecutively, the patients with recurrent tumors may have a short period of no treatment 
after surgery and chemotherapy. This period may have contributed to the patient's functional recovery and enhanced the patient's tolerance to chemotherapy. Thirdly, different subsequent treatments might have an impact on patients' survival.

Unfortunately, the sudden spread of the new coronavirus (SARS-CoV-2) and resulting disease (COVID19) impacts our existing medical system and limited medical resources. Many patients have delayed the treatment of tumors due to the epidemic. Our research protocol shows a unique advantage, that is, it can provide patients with a continued systemic treatment option without a suspension or delay. A national analysis of COVID-19 in China suggested that cancer patients may be at relatively higher risk of infection with novel coronavirus and severe events[28]. This protocol not only achieves psychological and physical requirements of advanced gastric cancer patients, but might also help to reduce the spread of COVID-19 indirectly through reducing the need of hospital visits and exposure.

In summary, this study has certain advantages: Firstly, our treatment regimen effectively improved the disease control rate $(83.8 \%$ ), and improved survival in patients with advanced gastric or gastro-oesophageal junction adenocarcinoma refractory to first-line platinum-containing treatment. Secondly, we explored a safe and effective home-based treatment option, which might benefit both the susceptible patients and spare limited medical resources during the COVID-19 epidemic. Thirdly, despite the high incidence of adverse events, compared with intravenous chemotherapy, the toxicity of oral targeted drug combined with oral chemotherapy drug is milder and reasonably manageable. Because of the good tolerance, there were no treatment-related deaths in our study. Fourthly, this trial explored a regimen in elder patients and those with poor physical performance, for instance, an 84-year-old patient was included in this study and reached partial response after 4 cycles of treatment. At the data-cutoff, this patient continued to receive the treatment with apatinib plus S-1, with a PFS of more than 8.8 months.

Conversely, our study also has several limitations. In the absence of randomization, the selection bias could not be trimmed, therefore, the strength of the medical evidence was not sufficient. Because of the small number of patients enrolled, our findings might be affected by the sampling error. In addition, because of a large number of new drugs appearing in recent years, considering individual patients' requirements, the wide treatment choices made enrollment difficult, resulting in a longer time span for enrollment in this study. However, encouragingly, the enrolled patients maintained a good compliance to this trial.

In conclusion, the efficacy of apatinib plus S-1 was comparable to the existing research, and the safety profile seemed to be more favorable than that of intravenous treatment regimens. Furthermore, our study provides a new option for the second-line treatment for advanced gastric or gastro-oesophageal junction adenocarcinoma, and explores a low-toxic and more convenient medication regimen, especially for elderly patients with poor condition. In addition, apatinib plus S-1 may be an appropriate choice of second-line treatment for advanced gastric cancer during the COVID-19 pandemic.

\section{Acknowledgments}

This study was supported by National Key Technologies R\&D Program (No. 2015BAI13B09) and China Anti-Cancer Association-Hengrui Pharmaceutical Innovative Drugs Clinical Research Fund. We thank all the patients and their families and all the participating staffs.

\section{Conflict of interest}

The authors declare no potential conflicts of interest.

\section{Author Contributions}

Conception and design: Wei Deng, Zhongtao Zhang

Financial support: Wei Deng

Administrative support: Zhongtao Zhang, Jun Zhang, Wei Deng. 
Provision of study materials and patients: Wei Deng, Zhigang Bai, Zhongtao Zhang, Jun Zhang, Jie Yin, Jun Cai

Collection and assembly of data: Chao Jing, Hongpeng Jiang, Xiaobao Yang, Kai Pang, Shu Yan

Data analysis and interpretation: Chao Jing, Wei Deng

Manuscript writing: Chao Jing, Wei Deng

Final approval of manuscript: all authors

Accountable for all aspects of the work: all authors

Data availability statement:

The data that support the findings of this study are available from the corresponding author upon reasonable request.

\section{References}

1. Bray F, Ferlay J, Soerjomataram I, Siegel RL, Torre LA, Jemal A. Global cancer statistics 2018: GLOBOCAN estimates of incidence and mortality worldwide for 36 cancers in 185 countries. CA Cancer J Clin. 2018;68(6):394-424.

2. Siegel RL, Miller KD, Jemal A. Cancer statistics, 2020. CA Cancer J Clin. 2020;70(1):7-30.

3. Suh YS, Yang HK. Screening and Early Detection of Gastric Cancer: East Versus West. Surg Clin North Am. 2015;95(5):1053-1066.

4. Leung WK, Wu MS, Kakugawa Y, Kim JJ, Yeoh KG, Goh KL, et al. Screening for gastric cancer in Asia: current evidence and practice.Lancet Oncol. 2008;9(3):279-287.

5. Khanderia E, Markar SR, Acharya A, Kim Y, Kim YW, Hanna GB. The Influence of Gastric Cancer Screening on the Stage at Diagnosis and Survival: A Meta-Analysis of Comparative Studies in the Far East.J Clin Gastroenterol. 2016;50(3):190-197.

6. Zong L, Abe M, Seto Y, Ji J. The challenge of screening for early gastric cancer in China. Lancet. 2016;388(10060):2606.

7. Noh SH, Park SR, Yang HK, Chung HC, Chung IJ, Kim SW, et al. Adjuvant capecitabine plus oxaliplatin for gastric cancer after D2 gastrectomy (CLASSIC): 5-year follow-up of an open-label, randomised phase 3 trial.Lancet Oncol. 2014;15(12):1389-1396.

8. Ajani JA, D'Amico TA, Almhanna K, Bentrem DJ, Chao J, Das P, et al. Gastric Cancer, Version 3.2016, NCCN Clinical Practice Guidelines in Oncology. J Natl Compr Canc Netw. 2016;14(10):1286-1312.

9. Wang FH, Shen L, Li J, Zhou ZW, Liang H, Zhang XT, et al. The Chinese Society of Clinical Oncology (CSCO): clinical guidelines for the diagnosis and treatment of gastric cancer. Cancer Commun (Lond).2019;39(1):10.

10. Park DJ, Thomas NJ, Yoon C, Yoon SS. Vascular endothelial growth factor a inhibition in gastric cancer. Gastric Cancer.2015;18(1):33-42.

11. Roviello G, Ravelli A, Polom K, Petrioli R, Marano L, Marrelli D, et al. Apatinib: A novel receptor tyrosine kinase inhibitor for the treatment of gastric cancer. Cancer Lett. 2016;372(2):187-191.

12. Li J, Qin S, Xu J, Guo W, Xiong J, Bai Y, et al. Apatinib for chemotherapy-refractory advanced metastatic gastric cancer: results from a randomized, placebo-controlled, parallel-arm, phase II trial. J Clin Oncol. 2013;31(26):3219-3225. 
13. Li J, Qin S, Xu J, Xiong J, Wu C, Bai Y, et al. Randomized, Double-Blind, Placebo-Controlled Phase III Trial of Apatinib in Patients With Chemotherapy-Refractory Advanced or Metastatic Adenocarcinoma of the Stomach or Gastroesophageal Junction. J Clin Oncol.2016;34(13):1448-1454.

14. van Groeningen CJ, Peters GJ, Schornagel JH, Gall H, Noordhuis P, de Vries MJ, et al. Phase I clinical and pharmacokinetic study of oral S-1 in patients with advanced solid tumors. J Clin Oncol.2000;18(14):27722779 .

15. Sakuramoto S, Sasako M, Yamaguchi T, Kinoshita T, Fujii M, Nashimoto A, et al. Adjuvant chemotherapy for gastric cancer with S-1, an oral fluoropyrimidine. N Engl J Med. 2007;357(18):1810-1820.

16. Kim GM, Jeung HC, Rha SY, Kim HS, Jung I, Nam BH, et al. A randomized phase II trial of S-1oxaliplatin versus capecitabine-oxaliplatin in advanced gastric cancer. Eur J Cancer. 2012;48(4):518-526.

17. Feng J, Qin S. The synergistic effects of Apatinib combined with cytotoxic chemotherapeutic agents on gastric cancer cells and in a fluorescence imaging gastric cancer xenograft model. Onco Targets Ther. 2018;11:3047-3057.

18. Schrag D, Hershman DL, Basch E. Oncology Practice During the COVID-19 Pandemic. JAMA. 2020.

19. Eisenhauer EA, Therasse P, Bogaerts J, Schwartz LH, Sargent D, Ford R, et al. New response evaluation criteria in solid tumours: revised RECIST guideline (version 1.1). Eur J Cancer. 2009;45(2):228-247.

20. Fuchs CS, Tomasek J, Yong CJ, Dumitru F, Passalacqua R, Goswami C, et al. Ramucirumab monotherapy for previously treated advanced gastric or gastro-oesophageal junction adenocarcinoma (REGARD): an international, randomised, multicentre, placebo-controlled, phase 3 trial. Lancet. 2014;383(9911):31-39.

21. Wilke H, Muro K, Van Cutsem E, Oh SC, Bodoky G, Shimada Y, et al. Ramucirumab plus paclitaxel versus placebo plus paclitaxel in patients with previously treated advanced gastric or gastro-oesophageal junction adenocarcinoma (RAINBOW): a double-blind, randomised phase 3 trial.Lancet Oncol. 2014;15(11):12241235 .

22. Hironaka S, Ueda S, Yasui H, Nishina T, Tsuda M, Tsumura T, et al. Randomized, open-label, phase III study comparing irinotecan with paclitaxel in patients with advanced gastric cancer without severe peritoneal metastasis after failure of prior combination chemotherapy using fluoropyrimidine plus platinum: WJOG 4007 trial. J Clin Oncol. 2013;31(35):4438-4444.

23. Shitara K, Ozguroglu M, Bang YJ, Di Bartolomeo M, Mandala M, Ryu MH, et al. Pembrolizumab versus paclitaxel for previously treated, advanced gastric or gastro-oesophageal junction cancer (KEYNOTE-061): a randomised, open-label, controlled, phase 3 trial. Lancet.2018;392(10142):123-133.

24. Takahari D. Second-line chemotherapy for patients with advanced gastric cancer. Gastric Cancer. 2017;20(3):395-406.

25. Puhr HC, Preusser M, Prager G, Ilhan-Mutlu A. New Treatment Options for Advanced Gastroesophageal Tumours: Mature for the Current Practice?Cancers (Basel). 2020;12(2).

26. Lan CY, Wang Y, Xiong Y, Li JD, Shen JX, Li YF, et al. Apatinib combined with oral etoposide in patients with platinum-resistant or platinum-refractory ovarian cancer (AEROC): a phase 2, single-arm, prospective study. Lancet Oncol. 2018;19(9):1239-1246.

27. Donskov F, Michaelson MD, Puzanov I, Davis MP, Bjarnason GA, Motzer RJ, et al. Sunitinib-associated hypertension and neutropenia as efficacy biomarkers in metastatic renal cell carcinoma patients. $\mathrm{Br} J$ Cancer. 2015;113(11):1571-1580.

28. Liang W, Guan W, Chen R, Wang W, Li J, Xu K, et al. Cancer patients in SARS-CoV-2 infection: a nationwide analysis in China. Lancet Oncol. 2020;21(3):335-337. 


\begin{tabular}{ll}
\hline All patients enrolled $(\mathrm{n}=37)$ & All patients enrolled $(\mathrm{n}=37)$ \\
\hline Age, years Median Range & $5928-84(\mathrm{IQR} 50-66)$ \\
Sex Male Female & $29(78 \%) 8(22 \%)$ \\
ECOG PS 012 & $7(19 \%) 25(68 \%) 5(13 \%)$ \\
Primary lesion Gastroesophageal junction Gastric & $9(24 \%) 28(76 \%)$ \\
Tumor status* Recurrent Unresectable & $14(38 \%) 22(62 \%)$ \\
No. Of metastatic sites >2 [?]2 & $9(24 \%) 28(76 \%)$ \\
Platinum-containing treatment duration >4months [?]4months & $16(43 \%) 21(57 \%)$ \\
\hline
\end{tabular}

Table 1. Baseline Demographic and Clinical Characteristics of Full Analysis Set. Data are presented as median

\begin{tabular}{lll}
\hline & Any grade & Grade 3-4 \\
\hline Non-hematological & $11(29.7 \%)$ & $1(2.7 \%)$ \\
Hypertension & $10(27.0 \%)$ & \\
Proteinuria & $12(32.4 \%)$ & $2(5.4 \%)$ \\
Hand-foot syndrome & $7(18.9 \%)$ & $1(2.7 \%)$ \\
Fatigue & $11(29.7 \%)$ & \\
Anorexia & $10(27.0 \%)$ & \\
Abdominal pain & $12(32.4 \%)$ & $1(2.7 \%)$ \\
Diarrhea & $6(16.2 \%)$ & \\
Dizziness & $14(37.8 \%)$ & \\
Elevated transaminase & $6(16.2 \%)$ & \\
Elevated bilirubin & $4(10.8 \%)$ & \\
Mucositis & & $1(2.7 \%)$ \\
Acute intestinal obstruction & & $1(2.7 \%)$ \\
Upper gastrointestinal bleeding & & \\
Hematological & $15(40.5 \%)$ & $1(2.7 \%)$ \\
Anemia & $12(32.4 \%)$ & $1(2.7 \%)$ \\
Leukopenia & $7(18.9 \%)$ & \\
Thrombocytopenia & & \\
\hline
\end{tabular}

Table 2. The incidence of adverse events in the safety population $(n=37)$.

$\overline{\text { Figure 1: Trial profile }}$

Figure 2: Kaplan-Meier curve for (A) overall survival and (B) progression-free survival in patients with at

Figure 3. Waterfall plot for the best percentage change in target lesions size This plot shows the best percentag

Figure 4: Kaplan-Meier curve for overall survival in patients with $(\mathrm{n}=14)$ or without radical gastrectomy 
Figure 1

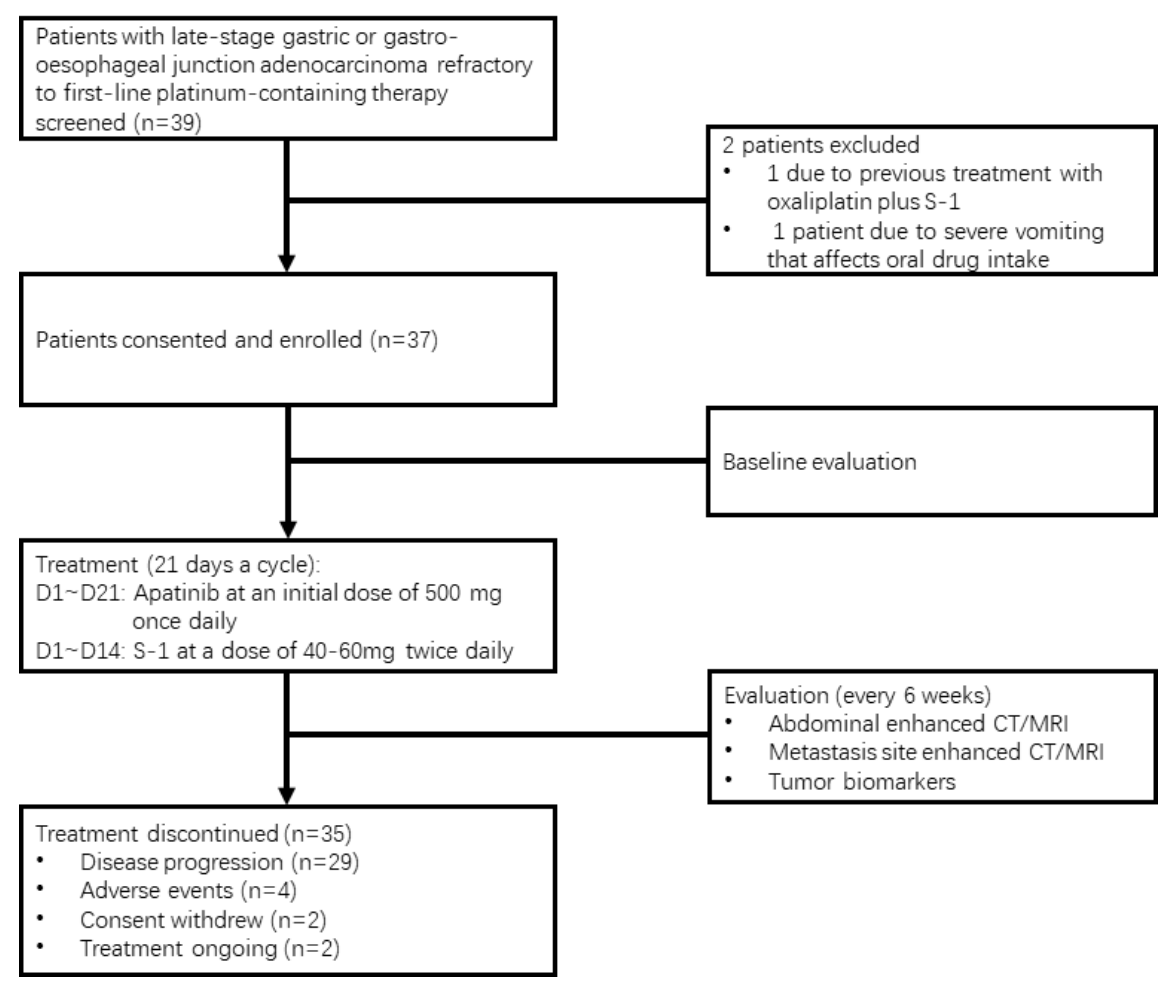

Figure 2
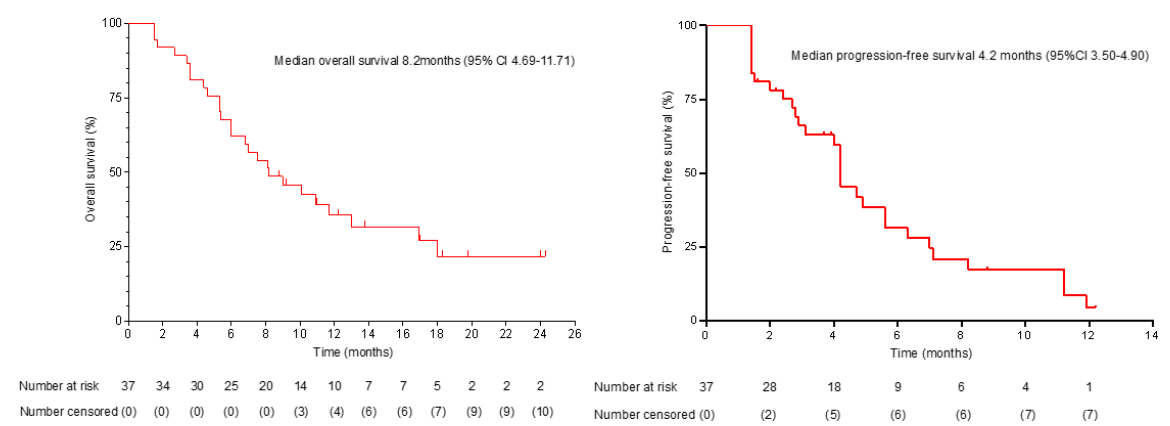

Number at nisk 37

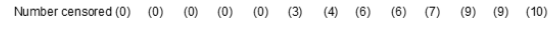
Number censored (0)

\section{1 due to previous treatment with oxaliplatin plus S-1 \\ 1 patient due to severe vomiting that affects oral drug intake}

valuation (every 6 weeks)

sis site enhanced CT/MR

Tumor biomarkers 
Figure 3

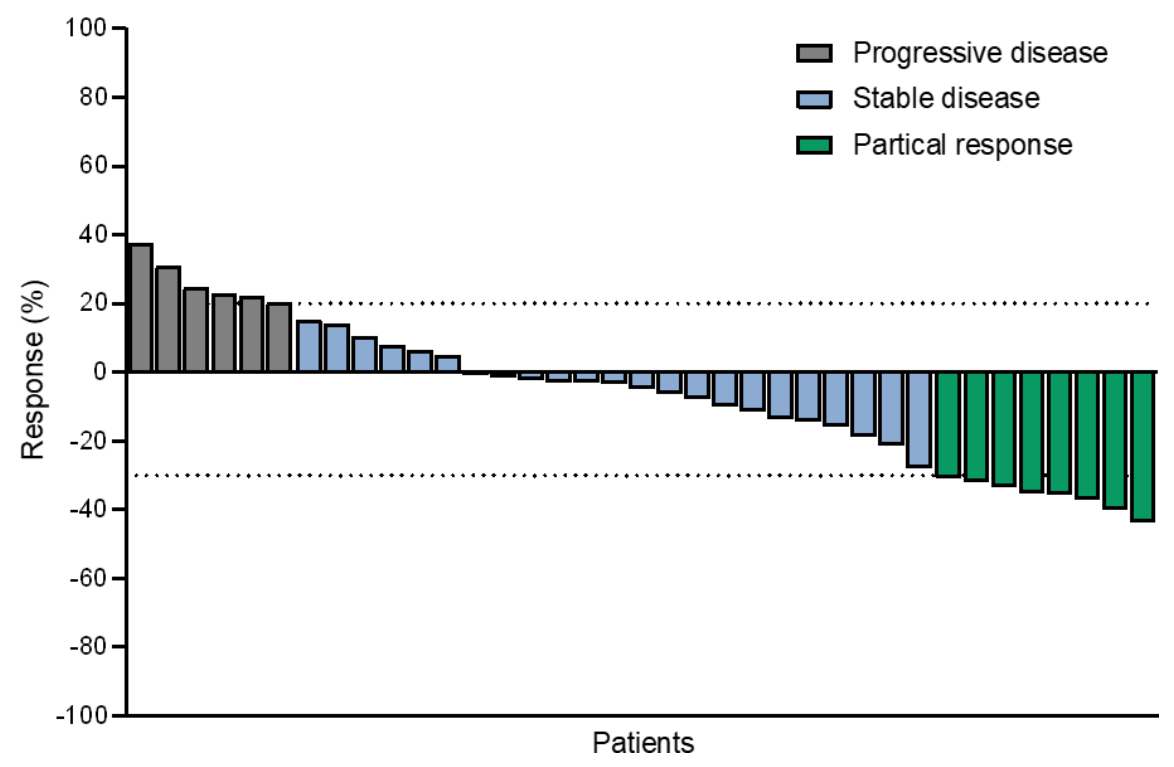

Figure 4

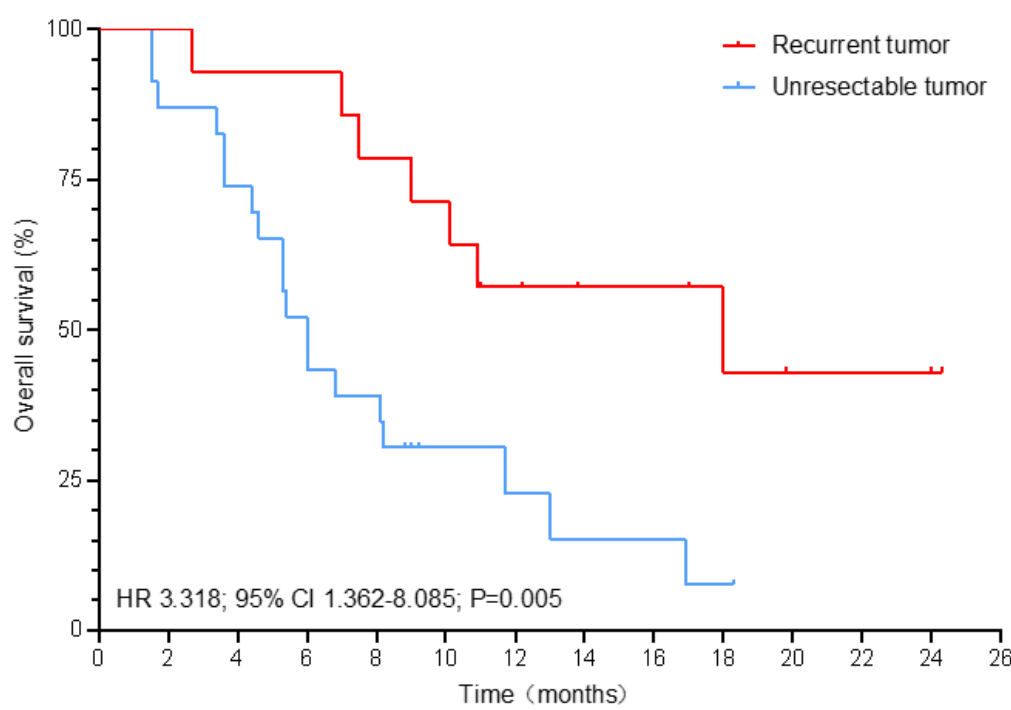

Number at risk

$\begin{array}{lccccccccccccc}\text { Recurrent tumor } & 14 & 14 & 13 & 13 & 11 & 10 & 7 & 5 & 5 & 4 & 2 & 2 & 2 \\ \text { Unresectable tumor } 23 & 20 & 17 & 12 & 9 & 4 & 3 & 2 & 2 & 1 & 0 & 0 & 0\end{array}$

\section{Hosted file}

Table 1.xlsx available at https://authorea.com/users/345804/articles/471912-apatinib-plus-s1-for-previously-treated-advanced-gastric-or-gastro-oesophageal-junction-adenocarcinomaa-phase-2-single-arm-prospective-study

\section{Hosted file}

Table 2.xlsx available at https://authorea.com/users/345804/articles/471912-apatinib-plus-s- 
1-for-previously-treated-advanced-gastric-or-gastro-oesophageal-junction-adenocarcinomaa-phase-2-single-arm-prospective-study 